See discussions, stats, and author profiles for this publication at: http://www.researchgate.net/publication/261749688

\title{
Assessment of the worldwide burden of critical illness: the Intensive Care Over Nations (ICON) audit
}

ARTICLE in THE LANCET RESPIRATORY MEDICINE · APRIL 2014

ImpactFactor: 9.63· DOI: 10.1016/S2213-2600(14)70061-X·Source: PubMed

CITATIONS

14

721 AUTHORS, INCLUDING:

Tarek Tantawy

Prince Sultan Cardiac Center

3 PUBLICATIONS 14 CITATIONS

SEE PROFILE

Ingo Voigt

Elisabeth-Krankenhaus, Essen

12 PUBLICATIONS 26 CITATIONS

SEE PROFILE
READS

551

Rubens Carmo Costa Filho

Hospital Pró-Cardíaco

58 PUBLICATIONS 228 CITATIONS

SEE PROFILE

Ulrich Jaschinski

Klinikum Augsburg

7 PUBLICATIONS 111 CITATIONS

SEE PROFILE 


\title{
(1) Assessment of the worldwide burden of critical illness: the Intensive Care Over Nations (ICON) audit
}

\author{
Jean-Louis Vincent, John C Marshall, Silvio A Ñamendys-Silva, Bruno François, Ignacio Martin-Loeches, Jeffrey Lipman, Konrad Reinhart, \\ Massimo Antonelli, Peter Pickkers, Hassane Njimi, Edgar Jimenez, Yasser Sakr, on behalf of the ICON investigators*
}

Lancet Respir Med 2014; 2: 380-86

Published Online April 14, 2014 http://dx.doi.org/10.1016/ S2213-2600(14)70061-X See Comment page 343 * Details of ICON investigators are given in the appendix Department of Intensive Care, Erasme University Hospital, Université Libre de Bruxelles, Brussels, Belgium (Prof)-LVincent MD, H Njimi PhD); Department of Surgery, Interdepartmental

Division of Critical Care Medicine, University of Toronto, St Michael's Hospital, Toronto, ON, Canada

(Prof J C Marshall MD); Department of Critical Care Medicine, Instituto Nacional de Cancerología, Mexico City, Mexico

(S A Ñamendys-Silva MD); Service de Réanimation Polyvalente, CHU Dupuytren, Limoges cedex, France (Prof B François MD); Critical Care Centre, Corporació Sanitària I Universitaria Parc Taulí-Hospital De Sabadell, Institut Universitari Uab, Ciber Enfermedades Respiratorias, Sabadell, Barcelona, Spain (I Martin-Loeches MD); Department of Intensive Care Medicine, Royal Brisbane and Women's Hospital, University of Queensland, QLD, Australia (Prof J Lipman MD); Department of Anaesthesiology and Intensive Care and Centre for Sepsis Care and Control, Jena University Hospital, Jena, Germany (Prof K Reinhart MD,

Y Sakr MD); Department of Anaesthesiology and Intensive Care, Catholic UniversityPoliclinico A Gemelli University Hospital, Rome, Italy (Prof M Antonelli MD); Department of Intensive Care, Nijmegen Institute for Infection, Inflammation and Immunity, Radboud University Nijmegen Medical Centre, Nijmegen, Netherlands (P Pickkers MD); and Department of Critical Care, Orlando

\section{Summary}

Background Global epidemiological data regarding outcomes for patients in intensive care units (ICUs) are scarce, but are important in understanding the worldwide burden of critical illness. We, therefore, did an international audit of ICU patients worldwide and assessed variations between hospitals and countries in terms of ICU mortality.

Methods 730 participating centres in 84 countries prospectively collected data on all adult (>16 years) patients admitted to their ICU between May 8 and May 18, 2012, except those admitted for fewer than $24 \mathrm{~h}$ for routine postoperative monitoring. Participation was voluntary. Data were collected daily for a maximum of 28 days in the ICU and patients were followed up for outcome data until death or hospital discharge. In-hospital death was analysed using multilevel logistic regression with three levels: patient, hospital, and country.

Findings 10069 patients were included from ICUs in Europe (5445 patients; 54.1\%), Asia (1928; 19.2\%), the Americas (1723; 17.1\%), Oceania (439; 4.4\%), the Middle East (393; 3.9\%), and Africa (141; 1.4\%). Overall, 2973 patients $(29 \cdot 5 \%)$ had sepsis on admission or during the ICU stay. ICU mortality rates were $16 \cdot 2 \%$ (95\% CI 15.5-16.9) across the whole population and $25 \cdot 8 \%(24 \cdot 2-27 \cdot 4)$ in patients with sepsis. Hospital mortality rates were $22.4 \%(21 \cdot 6-23 \cdot 2)$ in the whole population and $35 \cdot 3 \%(33 \cdot 5-37 \cdot 1)$ in patients with sepsis. Using a multilevel analysis, the unconditional model suggested significant between-country variations (var $=0 \cdot 19, p=0 \cdot 002)$ and betweenhospital variations $($ var $=0.43, p<0.0001)$ in the individual risk of in-hospital death. There was a stepwise increase in the adjusted risk of in-hospital death according to decrease in global national income.

Interpretation This large database highlights that sepsis remains a major health problem worldwide, associated with high mortality rates in all countries. Our findings also show a significant association between the risk of death and the global national income and suggest that ICU organisation has an important effect on risk of death.

Funding None.

\section{Introduction}

Intensive care medicine has grown substantially over the past decades and now consumes a substantial part of the income of many countries worldwide (close to $1 \%$ of the gross domestic product [GDP] in the USA ${ }^{1}$ ). Previous studies have provided some epidemiological data regarding types of patients and treatments used in intensive care units (ICUs) and outcomes for patients in ICUs at a local and a national level, but there is much less information available at an international level. ${ }^{2}$ A review in 2010 stressed that there is a "need to measure the global burden of critical illness and available critical-care resources, and develop both preventive and therapeutic interventions that are generalisable across countries". 'The World Federation of Societies of Intensive and Critical Care Medicine, with a membership of more than 70 national societies of intensive and critical care medicine, provided a unique platform to initiate an audit of data from ICUs around the world to develop an international picture of the types of critically ill patients admitted to our ICUs, with a special emphasis on sepsis and organ failure. We provide a summary of the key findings of this major worldwide collaborative initiative, providing important insights into characteristics of intensive care patient populations and variations in mortality rates between different countries and regions of the globe.

\section{Methods \\ Participating centres}

Recruitment for participation in the Intensive Care Over Nations (ICON) audit was by open invitation, through national scientific societies, national and international meetings, and individual contacts. Participation was entirely voluntary, with no financial incentive. Institutional review board approval was obtained by the participating institutions in accordance with local ethical regulations.

Each participating centre (appendix) was asked to prospectively collect data on all adult patients ( $>16$ years) admitted to their ICU between May 8 and May 18, 2012, except those who stayed in the ICU for fewer than $24 \mathrm{~h}$ for routine postoperative surveillance. Readmissions of previously included patients were excluded. Data were collected daily during the ICU stay for a maximum of 28 days. Patients were followed up for outcome data until death or hospital discharge.

Case report forms (CRFs; appendix) were electronically provided by the investigators using a secured internetbased website. Data collection on admission included 
demographic data and comorbid diseases. Clinical and laboratory data for simplified acute physiology score (SAPS) $\mathrm{II}^{3}$ and acute physiology and chronic health evaluation (APACHE) $\mathrm{II}^{4}$ scores were reported as the worst values within $24 \mathrm{~h}$ after admission. Microbiological and clinical infections were reported daily as well as the antibiotics given. A daily assessment of organ function in accordance with the sequential organ failure assessment (SOFA) score $^{5}$ was done.

\section{Definitions}

Infection was defined in accordance with the definitions of the International Sepsis Forum. ${ }^{6}$ Sepsis was defined as the presence of infection with the concomitant occurrence of at least one organ failure (defined as a SOFA score $>2$ for the organ in question). ${ }^{7}$ Septic shock was defined as sepsis associated with cardiovascular failure (defined as a cardiovascular SOFA score $>2$ ).

Surgical admissions referred to patients who had had surgery in the 4 weeks preceding admission. The presence of several comorbid disorders ${ }^{3,4}$ was noted: chronic obstructive pulmonary disease (COPD), metastatic cancer (metastases proven by surgery, CT or MRI, or any other method), liver cirrhosis, heart failure (New York Heart Association classification [NYHA] III/IV), haematological malignancy (lymphoma, acute leukaemia, or multiple myeloma), acquired immunodeficiency syndrome, chronic renal failure (need for chronic renal support or history of chronic renal insufficiency with a serum creatinine greater than $3.6 \mathrm{mg} / \mathrm{dL}[300 \mu \mathrm{mol} / \mathrm{L}]^{5}$ ), immunosuppression (steroid treatment given in the 6 months before ICU admission [at least $0.3 \mathrm{mg} / \mathrm{kg}$ per day prednisolone for at least 1 month], congenital immune-humoral, or cellular immune deficiency state), chemotherapy or radiotherapy (in the 6 months before ICU admission), severe malnutrition, and insulin-dependent diabetes mellitus.

\section{Data management and quality control}

Detailed instructions explaining the aim of the study, instructions for data collection, and definitions were available through a secured website for all participants before starting data collection and throughout the study period. Any additional queries were answered on a percase basis by the coordinating centre during data collection. Validity checks were made concurrent with data entry on the electronic CRF including plausibility checks within each variable and between variables. Data were further reviewed by the coordinating centre for plausibility and availability of outcome parameter (death in the ICU), and any doubts were clarified with the centre in question. There was no on-site monitoring.

\section{Statistical analysis}

Data were processed and analysed in the department of intensive care of the University of Brussels, in collaboration with the Jena University Hospital (Jena,
Germany). The appendix includes additional details of Regional Medical Center, the statistical analysis.

For the purposes of this study, the world was divided into nine geographical regions: North America, South America, western Europe, eastern Europe, Middle East, south Asia, east and southeast Asia, Oceania, and Africa. Individual countries were also classified into three income groups in accordance with their 2011 gross national income (GNI) per person, using thresholds defined by the

\begin{tabular}{|c|c|c|c|c|}
\hline & $\begin{array}{l}\text { All patients, } \\
\mathrm{n}=10069\end{array}$ & $\begin{array}{l}\text { Low and lower- } \\
\text { middle income, } \\
\mathrm{n}=1209\end{array}$ & $\begin{array}{l}\text { Upper-middle } \\
\text { income, } \\
\mathrm{n}=2504\end{array}$ & $\begin{array}{l}\text { High income, } \\
n=6356\end{array}$ \\
\hline Number of patients per centre & $10(5-18)$ & $10(4-29)$ & $8(4-13)$ & $11(5-20)$ \\
\hline \multicolumn{5}{|l|}{ Severity scores } \\
\hline SAPS II score & $40 \cdot 2(18 \cdot 2)$ & $33.4(17.5)^{*} \dagger$ & $40 \cdot 7(18 \cdot 0)$ & $41 \cdot 2(18 \cdot 1)$ \\
\hline APACHE II score & $17 \cdot 9(9 \cdot 4)$ & $14.3(8.9)^{*} \dagger$ & $17 \cdot 7(9 \cdot 4)^{*}$ & $18 \cdot 7(9 \cdot 3)$ \\
\hline SOFA score & $6 \cdot 3(4 \cdot 2)$ & $4 \cdot 6(4 \cdot 0)^{*} \dagger$ & $6 \cdot 0(4 \cdot 3)^{*}$ & $6 \cdot 3(4 \cdot 4)$ \\
\hline \multicolumn{5}{|l|}{ Type of admission } \\
\hline Surgical (non-trauma) & $3432(36.0 \%)$ & $317(28 \cdot 2 \%)^{*} \dagger$ & $926(39 \cdot 3 \%)^{*}$ & $218(36 \cdot 2 \%)$ \\
\hline Medical & $5382(56 \cdot 5 \%)$ & $728(64 \cdot 8 \%)^{*} \dagger$ & $1216(51 \cdot 6 \%)$ & $3438(56 \cdot 9 \%)$ \\
\hline Trauma & $643(6 \cdot 8 \%)$ & $71(6 \cdot 3 \%)$ & $189(8.0 \%)^{*}$ & $383(6 \cdot 3 \%)$ \\
\hline Other & $66(0.7 \%)$ & $7(0.6 \%)$ & $24(1.0 \%)$ & $35(0.6 \%)$ \\
\hline \multicolumn{5}{|l|}{ Source of admission } \\
\hline Emergency room or ambulance & $3814(37 \cdot 9 \%)$ & $438(36 \cdot 2 \%)$ & $918(36 \cdot 7 \%)$ & $2458(38.7 \%)$ \\
\hline Hospital floor & $2625(26 \cdot 1 \%)$ & $221(18 \cdot 3 \%)^{*} \dagger$ & $773(30 \cdot 9 \%)^{*}$ & $1631(25 \cdot 7 \%)$ \\
\hline Operating room or recovery room & $1811(18 \cdot 0 \%)$ & $147(12 \cdot 2 \%)^{*} \dagger$ & $423(16 \cdot 9 \%)^{*}$ & $1241(19 \cdot 5 \%)$ \\
\hline Other hospital & $981(9 \cdot 7 \%)$ & $165(13 \cdot 6 \%)^{*} \dagger$ & $242(9 \cdot 7 \%)$ & $574(9 \cdot 0 \%)$ \\
\hline Other & $838(8 \cdot 3 \%)$ & $238(19 \cdot 7 \%)^{*} \dagger$ & $148(5 \cdot 9 \%)$ & $452(7 \cdot 1 \%)$ \\
\hline \multicolumn{5}{|l|}{ Comorbidities } \\
\hline COPD & $1240(12 \cdot 3 \%)$ & $72(6 \cdot 0 \%)^{*} \dagger$ & $268(10 \cdot 7 \%)^{*}$ & $900(14 \cdot 2 \%)$ \\
\hline Cancer (solid, non-metastatic) & $888(8 \cdot 8 \%)$ & $73(6 \cdot 0 \%)^{*}$ & $183(7 \cdot 3 \%)^{*}$ & $632(9 \cdot 9 \%)$ \\
\hline Diabetes mellitus, insulin-dependent & $972(9 \cdot 7 \%)$ & $129(10 \cdot 7 \%)$ & $219(8 \cdot 7 \%)$ & $624(9 \cdot 8 \%)$ \\
\hline Heart failure, NYHA III/IV & $921(9 \cdot 1 \%)$ & $64(5 \cdot 3 \%)^{*} \dagger$ & $292(11 \cdot 7 \%)^{*}$ & $565(8 \cdot 9 \%)$ \\
\hline Chronic renal failure & $912(9 \cdot 1 \%)$ & $80(6 \cdot 6 \%)^{*}$ & $188(7 \cdot 5 \%)^{*}$ & $644(10 \cdot 1 \%)$ \\
\hline Immunosuppression & $757(7.5 \%)$ & $63(5 \cdot 2 \%)^{*} \dagger$ & $168(6 \cdot 7 \%)$ & $526(8 \cdot 3 \%)$ \\
\hline Cirrhosis & $349(3 \cdot 5 \%)$ & $27(2 \cdot 2 \%)^{*}$ & $78(3 \cdot 1 \%)$ & $244(3 \cdot 8 \%)$ \\
\hline Metastatic cancer & $332(3 \cdot 3 \%)$ & $33(2 \cdot 7 \%)$ & $70(2 \cdot 8 \%)$ & $229(3 \cdot 6 \%)$ \\
\hline Haematological cancer & $212(2 \cdot 1 \%)$ & $11(0.9 \%)^{*}$ & $38(1.5 \%)^{*}$ & $163(2 \cdot 6 \%)$ \\
\hline HIV infection & $71(0.7 \%)$ & $3(0 \cdot 2 \%)$ & $24(1.0 \%)$ & $44(0.7 \%)$ \\
\hline \multicolumn{5}{|l|}{ Number of comorbidities } \\
\hline None & $5512(54 \cdot 7 \%)$ & $784(64 \cdot 8 \%)^{*} \dagger$ & $1396(55 \cdot 8 \%)^{*}$ & $3332(52 \cdot 4 \%)$ \\
\hline 1 & $2917(29 \cdot 0 \%)$ & $315(26 \cdot 1 \%)$ & $755(30 \cdot 2 \%)$ & $1847(29 \cdot 1 \%)$ \\
\hline 2 & $1252(12 \cdot 4 \%)$ & $92(7 \cdot 6 \%)^{*} \dagger$ & $289(11 \cdot 5 \%)^{*}$ & $871(13 \cdot 7 \%)$ \\
\hline 3 & $328(3 \cdot 3 \%)$ & $16(1 \cdot 3 \%)^{*}$ & $61(2 \cdot 4 \%)^{*}$ & $251(3 \cdot 9 \%)$ \\
\hline$\geq 4$ & $60(0.6 \%)$ & $2(0 \cdot 2 \%)$ & $3(0 \cdot 1 \%)$ & $55(0.9 \%)$ \\
\hline \multicolumn{5}{|l|}{ Infectious status } \\
\hline Infection & $2473(24 \cdot 6 \%)$ & $186(15 \cdot 4 \%)^{*} \dagger$ & $706(28 \cdot 2 \%)^{*}$ & $1581(24.9 \%)$ \\
\hline Sepsis & $1808(18.0 \%)$ & $120(9.9 \%)^{*} \dagger$ & $497(19 \cdot 8 \%)$ & $1191(18 \cdot 7 \%)$ \\
\hline Septic shock & $986(9 \cdot 8 \%)$ & $60(5.0 \%) \dagger^{*}$ & $227(9 \cdot 1 \%)^{*}$ & 699 (11.0\%) \\
\hline
\end{tabular}

Data are median (IQR), mean (SD), or $\mathrm{n}(\%)$. Valid percentages are given after exclusion of missing values (data missing from 546 patients for type of admission). SAPS=simplified acute physiology score. APACHE=acute physiology and chronic health evaluation. SOFA=sequential organ failure score. $C O P D=$ chronic obstructive pulmonary disease. NYHA=New York Heart Association classification. Statistically significant at $5 \%$ with Bonferroni correction: ${ }^{*} v$ h high. tvs upper middle.

Table 1: Characteristics of the study cohort on admission to the ICU by GNI stratification 
World Bank Atlas method:8 GNI less than US\$4035 was defined as low and lower-middle income, \$4036-\$12475 was defined as upper-middle income, and greater than $\$ 12476$ was defined as high income (appendix).

Data are summarised with means and SDs, medians and IQRs, or numbers and percentages. Crude mortality rates are given as percentages with Wald 95\% CIs. Single missing values of the SOFA score were imputed by linear interpolation. When first or last values were missing, the nearest value was carried backward or forward, respectively.

The Kolmogorov-Smirnov test was used, and histograms and quantile-quantile plots were examined to verify if there were significant deviations from the normality assumption of continuous variables. Difference testing between groups was done with ANOVA, Kruskal-Wallis test, Student's $t$ test, Mann-Whitney test, $\chi^{2}$ test, or Fisher's exact test, as appropriate. The least significant difference testing procedure was used for pairwise comparisons.

In-hospital death was analysed using multilevel logistic regression with three levels: patient, hospital, and country. The results of fixed effects (measures of association) are given as odds ratios (OR) with their 95\% CIs and the $80 \%$ interval OR. ${ }^{10-12}$ Random effects (measures of variation) measures included the variance (var) and its $\mathrm{SE}$, the proportional change in variance, ${ }^{12}$ and the median OR. ${ }^{10-12}$ The statistical significance of covariates were calculated with the Wald test. ${ }^{13}$

Data were analysed with IBM SPSS statistics software, version 20 for windows, and MLwiN, version 2.28. All reported $\mathrm{p}$ values are two-sided and a $\mathrm{p}$ value of less than 0.05 was deemed to show statistical significance.

\section{Role of the funding source}

There was no funding source for this study. The corresponding author had full access to all the data in the study and had final responsibility for the decision to submit for publication.

\section{Results}

10069 patients were included in the audit, most commonly from Europe (5445 patients; 54.1\%), Asia (1928; 19.2\%), and the Americas (1723; 17.1\%). Table 1 lists the characteristics of the audit cohort on admission to the ICU according to GNI. Patients admitted to ICUs in countries with lower GNI were less severely ill than those admitted in higher income countries; they were more likely to be medical patients and less likely to have comorbid COPD or heart failure (table 1). Table 2 shows the organisational characteristics of the participating centres-most ICUs were located in university or academic hospitals. The highest hospital bed capacities were in centres from countries with higher GNI. There were no other major organisational differences between the centres according to GNI.

Patients from low-income countries were less likely to receive mechanical ventilation or renal replacement therapy during the ICU stay than patients in uppermiddle or high income countries (all $\mathrm{p}<0 \cdot 0001$; table 3 ).

Almost a third of patients had sepsis during the ICU stay, but substantially lower occurrence rates were reported

\begin{tabular}{|c|c|c|c|c|}
\hline & All centres, $n=730$ & $\begin{array}{l}\text { Low and lower- } \\
\text { middle income, } n=62\end{array}$ & $\begin{array}{l}\text { Upper-middle } \\
\text { income, } n=237\end{array}$ & High income, $n=431$ \\
\hline Number of countries & 84 & 17 & 27 & 40 \\
\hline \multicolumn{5}{|l|}{ Type of hospital } \\
\hline University or academic & $419(57 \cdot 4 \%)$ & $26(41 \cdot 9 \%)^{*}$ & $148(62 \cdot 4 \%) \dagger$ & $245(56 \cdot 8 \%)$ \\
\hline Non-university & $247(33 \cdot 8 \%)$ & $27(43 \cdot 5 \%)^{*}$ & $62(26 \cdot 2 \%) \dagger$ & $158(36 \cdot 7 \%)$ \\
\hline Unknown & $64(8 \cdot 8 \%)$ & $9(14.5 \%)$ & $27(11 \cdot 4 \%)$ & $28(6 \cdot 5 \%)$ \\
\hline Hospital bed capacity & $600(320-982)$ & $352(200-600)^{*} \dagger$ & $550(200-1200)$ & $642(400-950)$ \\
\hline \multicolumn{5}{|l|}{ ICU specialty } \\
\hline Surgical & $83(11 \cdot 4 \%)$ & $7(11 \cdot 3 \%)$ & $26(11 \cdot 0 \%)$ & $50(11 \cdot 6 \%)$ \\
\hline Medical & $73(10 \cdot 0 \%)$ & $4(6 \cdot 5 \%)$ & $23(9 \cdot 7 \%)$ & $46(10 \cdot 7 \%)$ \\
\hline Mixed & $479(65 \cdot 6 \%)$ & $42(67 \cdot 7 \%)$ & $152(64 \cdot 1 \%)$ & $285(66 \cdot 1 \%)$ \\
\hline Others & $95(13 \cdot 0 \%)$ & $9(14 \cdot 5 \%)$ & $36(15 \cdot 2 \%)$ & $50(11 \cdot 6 \%)$ \\
\hline Number of ICU patients, 2011 & $700(429-1100)$ & $764(466-1405)$ & $624(386-1200)$ & $715(441-1082)$ \\
\hline ICU mortality rate, 2011 & $14(8-21)$ & $14(9-26)$ & $15(8-22)$ & $13(8-19)$ \\
\hline Number of staffed ICU beds, on the first day of the study & $12(8-18)$ & $12(10-20)$ & $14(10-18) \dagger$ & $12(8-16)$ \\
\hline ICU physician available $24 \mathrm{~h} / 24 \mathrm{~h}$ & $624(94 \cdot 5 \%)$ & $49(92 \cdot 5 \%)$ & $198(95 \cdot 2 \%)$ & $377(94 \cdot 5 \%)$ \\
\hline Physiotherapist available 24 h/24 h & $454(62 \cdot 2 \%)$ & $40(64 \cdot 5 \%)$ & $128(54 \%) \dagger$ & $286(66 \cdot 4 \%)$ \\
\hline Pharmacist available $24 \mathrm{~h} / 24 \mathrm{~h}$ & $276(37 \cdot 8 \%)$ & $23(37 \cdot 1 \%)$ & $84(35 \cdot 4 \%)$ & $169(39 \cdot 2 \%)$ \\
\hline Technician available $24 \mathrm{~h} / 24 \mathrm{~h}$ & $286(39 \cdot 2 \%)$ & $35(56 \cdot 5 \%) \dagger$ & $105(44 \cdot 3 \%) \dagger$ & $146(33 \cdot 9 \%)$ \\
\hline \multicolumn{5}{|c|}{$\begin{array}{l}\text { Data are } \mathrm{n}(\%) \text { or median (IQR). Valid percentages are displayed after exclusion of missing values (data missing from } 70 \text { centres for ICU physician availability, } 81 \text { centres for } \\
\text { number of ICU patients [2011], } 89 \text { centres for } 2011 \text { ICU mortality rates, } 68 \text { centres for number of staffed ICU beds, and } 105 \text { centres for hospital bed capacity). GNI=gross } \\
\text { national income. ICU=intensive care unit. Statistically significant at } 5 \% \text { with Bonferroni correction: *vs upper middle. †vs high. }\end{array}$} \\
\hline
\end{tabular}




\begin{tabular}{|c|c|c|c|c|c|c|c|c|c|c|c|c|}
\hline & \multirow{2}{*}{$\begin{array}{l}\text { Number } \\
\text { of } \\
\text { centres }\end{array}$} & \multirow{2}{*}{$\begin{array}{l}\text { Number } \\
\text { of patients } \\
\text { (\%) }\end{array}$} & \multirow[t]{2}{*}{$\begin{array}{l}\text { Mean age, } \\
\text { years (SD) }\end{array}$} & \multirow{2}{*}{$\begin{array}{l}\text { Mean } \\
\text { SAPS II } \\
\text { score } \\
\text { (SD) }\end{array}$} & \multirow{2}{*}{$\begin{array}{l}\text { Mean } \\
\text { APACHE II } \\
\text { score } \\
\text { (SD) }\end{array}$} & \multirow{2}{*}{$\begin{array}{l}\text { Number } \\
\text { of cases of } \\
\text { sepsis (\%) }\end{array}$} & \multicolumn{2}{|c|}{ Mortality rate, \% ( $95 \% \mathrm{Cl})$} & \multicolumn{2}{|c|}{$\begin{array}{l}\text { Median length } \\
\text { of stay, days (IQR) }\end{array}$} & \multirow{2}{*}{$\begin{array}{l}\text { Number of } \\
\text { patients } \\
\text { receiving } \\
\text { mechanical } \\
\text { ventilation (\%) }\end{array}$} & \multirow{2}{*}{$\begin{array}{l}\text { Number of } \\
\text { patients } \\
\text { receiving RRT } \\
(\%)\end{array}$} \\
\hline & & & & & & & ICU & In-hospital & ICU & In-hospital & & \\
\hline Total & 730 & 10069 & $\begin{array}{l}60 \\
(18)\end{array}$ & $\begin{array}{l}40 \cdot 2 \\
(18 \cdot 2)\end{array}$ & $\begin{array}{l}17 \cdot 9 \\
(9 \cdot 4)\end{array}$ & $\begin{array}{l}2973 \\
(29 \cdot 5 \%)\end{array}$ & $\begin{array}{l}16 \cdot 2 \\
(15 \cdot 5-16 \cdot 9)\end{array}$ & $\begin{array}{l}22 \cdot 4 \\
(21 \cdot 6-23 \cdot 2)\end{array}$ & $\begin{array}{l}3 \\
(2-6)\end{array}$ & $\begin{array}{l}10 \\
(5-20)\end{array}$ & $\begin{array}{l}5407 \\
(53 \cdot 7 \%)\end{array}$ & $1229(12 \cdot 2 \%)$ \\
\hline \multicolumn{13}{|l|}{ Region } \\
\hline Western Europe & 317 & $\begin{array}{r}4335 \\
(43 \cdot 1 \%)\end{array}$ & $\begin{array}{l}63 \\
(17)\end{array}$ & $\begin{array}{l}41 \cdot 7 \\
(18 \cdot 1)\end{array}$ & $\begin{array}{l}18 \cdot 8 \\
(9 \cdot 2)\end{array}$ & $\begin{array}{l}1357 \\
(31 \cdot 3 \%)\end{array}$ & $\begin{array}{l}15 \cdot 5 \\
(14 \cdot 4-16 \cdot 6)\end{array}$ & $\begin{array}{l}22 \cdot 6 \\
(21 \cdot 3-23 \cdot 9)\end{array}$ & $\begin{array}{l}3 \\
(1-6)\end{array}$ & $\begin{array}{l}11 \\
(6-22)\end{array}$ & $\begin{array}{l}2514 \\
(58 \cdot 0 \%)\end{array}$ & $553(12 \cdot 8 \%)$ \\
\hline Eastern Europe & 87 & $\begin{array}{r}1110 \\
(11.0 \%)\end{array}$ & $\begin{array}{l}60 \\
(17)\end{array}$ & $\begin{array}{l}41 \cdot 2 \\
(18 \cdot 2)\end{array}$ & $\begin{array}{l}18 \cdot 0 \\
(9 \cdot 4)\end{array}$ & $\begin{array}{c}336 \\
(30 \cdot 3 \%)\end{array}$ & $\begin{array}{l}21 \cdot 9 \\
(19 \cdot 5-24 \cdot 3)\end{array}$ & $\begin{array}{l}27 \cdot 2 \\
(24 \cdot 5-29 \cdot 9)\end{array}$ & $\begin{array}{l}3 \\
(2-7)\end{array}$ & $\begin{array}{l}10 \\
(6-18)\end{array}$ & $\begin{array}{c}651 \\
(58.6 \%)\end{array}$ & $113(10 \cdot 2 \%)$ \\
\hline South America & 109 & $\begin{array}{c}993 \\
(9.9 \%)\end{array}$ & $\begin{array}{l}59 \\
(20)\end{array}$ & $\begin{array}{l}40.8 \\
(18.8)\end{array}$ & $\begin{array}{l}17 \cdot 1 \\
(9 \cdot 4)\end{array}$ & $\begin{array}{c}303 \\
(30 \cdot 5 \%)\end{array}$ & $\begin{array}{l}21 \cdot 7 \\
(19 \cdot 0-24 \cdot 4)\end{array}$ & $\begin{array}{l}29 \cdot 4 \\
(26 \cdot 2-32 \cdot 6)\end{array}$ & $\begin{array}{l}4 \\
(2-7)\end{array}$ & $\begin{array}{c}9 \\
(5-20)\end{array}$ & $\begin{array}{c}509 \\
(51 \cdot 3 \%)\end{array}$ & $127(12 \cdot 8 \%)$ \\
\hline North America & 23 & $\begin{array}{l}730 \\
(7 \cdot 2 \%)\end{array}$ & $\begin{array}{l}59 \\
(18)\end{array}$ & $\begin{array}{l}35 \cdot 9 \\
(16 \cdot 5)\end{array}$ & $\begin{array}{l}17 \cdot 0 \\
(8 \cdot 4)\end{array}$ & $\begin{array}{c}147 \\
(20 \cdot 1 \%)\end{array}$ & $\begin{array}{c}9 \cdot 3 \\
(7 \cdot 2-11 \cdot 4)\end{array}$ & $\begin{array}{l}13 \cdot 1 \\
(10 \cdot 6-15 \cdot 6)\end{array}$ & $\begin{array}{l}2 \\
(1-4)\end{array}$ & $\begin{array}{c}6 \\
(3-14)\end{array}$ & $\begin{array}{c}267 \\
(36 \cdot 6 \%)\end{array}$ & $60(8 \cdot 2 \%)$ \\
\hline East and southeast Asia & 91 & $\begin{array}{r}946 \\
(9 \cdot 4 \%)\end{array}$ & $\begin{array}{l}60 \\
(18)\end{array}$ & $\begin{array}{l}43 \cdot 2 \\
(17 \cdot 2)\end{array}$ & $\begin{array}{l}19 \cdot 8 \\
(9 \cdot 6)\end{array}$ & $\begin{array}{c}372 \\
(39 \cdot 3 \%)\end{array}$ & $\begin{array}{l}16 \cdot 6 \\
(14 \cdot 2-19 \cdot 0)\end{array}$ & $\begin{array}{l}23 \cdot 7 \\
(20 \cdot 9-26 \cdot 5)\end{array}$ & $\begin{array}{l}4 \\
(2-7)\end{array}$ & $\begin{array}{l}11 \\
(5-25)\end{array}$ & $\begin{array}{c}571 \\
(60 \cdot 4 \%)\end{array}$ & $150(15 \cdot 9 \%)$ \\
\hline South Asia & 36 & $\begin{array}{r}982 \\
(9.8 \%)\end{array}$ & $\begin{array}{l}55 \\
(17)\end{array}$ & $\begin{array}{l}31 \cdot 3 \\
(16 \cdot 8)\end{array}$ & $\begin{array}{l}13 \cdot 2 \\
(8 \cdot 4)\end{array}$ & $\begin{array}{c}134 \\
(13 \cdot 6 \%)\end{array}$ & $\begin{array}{l}10 \cdot 9 \\
(8 \cdot 9-12 \cdot 9)\end{array}$ & $\begin{array}{l}14 \cdot 4 \\
(12 \cdot 0-16 \cdot 8)\end{array}$ & $\begin{array}{l}2 \\
(1-4)\end{array}$ & $\begin{array}{l}6 \\
(2-10)\end{array}$ & $\begin{array}{c}317 \\
(32 \cdot 3 \%)\end{array}$ & $73(7 \cdot 4 \%)$ \\
\hline Oceania & 20 & $\begin{array}{r}439 \\
(4 \cdot 4 \%)\end{array}$ & $\begin{array}{l}58 \\
(18)\end{array}$ & $\begin{array}{l}41 \cdot 2 \\
(14 \cdot 7)\end{array}$ & $\begin{array}{l}18 \cdot 5 \\
(7 \cdot 7)\end{array}$ & $\begin{array}{c}135 \\
(30 \cdot 8 \%)\end{array}$ & $\begin{array}{l}10 \cdot 3 \\
(7 \cdot 5-13 \cdot 1)\end{array}$ & $\begin{array}{l}13 \cdot 8 \\
(10 \cdot 6-17 \cdot 0)\end{array}$ & $\begin{array}{l}2 \\
(1-5)\end{array}$ & $\begin{array}{c}8 \\
(4-17)\end{array}$ & $\begin{array}{c}256 \\
(58 \cdot 3 \%)\end{array}$ & $45(10 \cdot 3 \%)$ \\
\hline Middle East & 36 & $\begin{array}{c}393 \\
(3.9 \%)\end{array}$ & $\begin{array}{l}55 \\
(20)\end{array}$ & $\begin{array}{l}42 \cdot 1 \\
(20 \cdot 8)\end{array}$ & $\begin{array}{l}19 \cdot 7 \\
(11 \cdot 2)\end{array}$ & $\begin{array}{c}151 \\
(38 \cdot 4 \%)\end{array}$ & $\begin{array}{l}26 \cdot 2 \\
(21 \cdot 8-30 \cdot 6)\end{array}$ & $\begin{array}{l}34 \cdot 1 \\
(29 \cdot 3-38 \cdot 9)\end{array}$ & $\begin{array}{l}4 \\
(2-9)\end{array}$ & $\begin{array}{l}10 \\
(5-23)\end{array}$ & $\begin{array}{c}252 \\
(64 \cdot 1 \%)\end{array}$ & $76(19 \cdot 3 \%)$ \\
\hline Africa & 11 & $\begin{array}{c}141 \\
(1 \cdot 4 \%)\end{array}$ & $\begin{array}{l}48 \\
(19)\end{array}$ & $\begin{array}{l}36 \cdot 1 \\
(17 \cdot 4)\end{array}$ & $\begin{array}{l}15 \cdot 3 \\
(9 \cdot 2)\end{array}$ & $\begin{array}{c}38 \\
(27 \cdot 0 \%)\end{array}$ & $\begin{array}{l}16 \cdot 9 \\
(10 \cdot 5-23 \cdot 3)\end{array}$ & $\begin{array}{l}20 \cdot 7 \\
(13 \cdot 3-28 \cdot 1)\end{array}$ & $\begin{array}{l}2 \\
(1-5)\end{array}$ & $\begin{array}{c}8 \\
(3-16)\end{array}$ & $\begin{array}{c}70 \\
(49 \cdot 6 \%)\end{array}$ & $32(22 \cdot 7 \%)$ \\
\hline \multicolumn{13}{|l|}{ GNI } \\
\hline $\begin{array}{l}\text { Low and lower-middle } \\
\text { income }\end{array}$ & 62 & $\begin{array}{r}1209 \\
(12 \cdot 0 \%)\end{array}$ & $\begin{array}{l}55 \\
(17)\end{array}$ & $\begin{array}{c}33 \cdot 4 \\
(17 \cdot 5)\end{array}$ & $\begin{array}{l}14 \cdot 3 \\
(8 \cdot 9)\end{array}$ & $\begin{array}{c}198 \\
(16 \cdot 4 \%)\end{array}$ & $\begin{array}{l}14 \cdot 1 \\
(13 \cdot 0-15 \cdot 1)\end{array}$ & $\begin{array}{l}18 \cdot 2 \\
(17 \cdot 0-19 \cdot 4)\end{array}$ & $\begin{array}{l}2 \\
(1-4)\end{array}$ & $\begin{array}{c}6 \\
(2-10)\end{array}$ & $\begin{array}{c}432 \\
(35 \cdot 7 \%)\end{array}$ & $87(7 \cdot 2 \%)$ \\
\hline Upper-middle income & 237 & $\begin{array}{c}2504 \\
(24 \cdot 9 \%)\end{array}$ & $\begin{array}{l}58 \\
(19)\end{array}$ & $\begin{array}{l}40.7 \\
(18.0)\end{array}$ & $\begin{array}{l}17 \cdot 7 \\
(9 \cdot 4)\end{array}$ & $\begin{array}{c}790 \\
(31 \cdot 5 \%)\end{array}$ & $\begin{array}{l}21 \cdot 4 \\
(20 \cdot 3-22 \cdot 2)\end{array}$ & $\begin{array}{l}27 \cdot 5 \\
(26 \cdot 6-28 \cdot 5)\end{array}$ & $\begin{array}{l}4 \\
(2-7)\end{array}$ & $\begin{array}{l}10 \\
(5-19)\end{array}$ & $\begin{array}{l}1377 \\
(55 \cdot 0 \%)\end{array}$ & $349(13 \cdot 9 \%)$ \\
\hline High income & 431 & $\begin{array}{r}6356 \\
(63 \cdot 1 \%)\end{array}$ & $\begin{array}{l}62 \\
(18)\end{array}$ & $\begin{array}{l}41 \cdot 2 \\
(18 \cdot 1)\end{array}$ & $\begin{array}{l}18 \cdot 7 \\
(9 \cdot 3)\end{array}$ & $\begin{array}{l}1985 \\
(31 \cdot 2 \%)\end{array}$ & $\begin{array}{l}14 \cdot 6 \\
(13 \cdot 8-15 \cdot 5)\end{array}$ & $\begin{array}{l}21 \cdot 2 \\
(20 \cdot 7-21 \cdot 8)\end{array}$ & $\begin{array}{l}3 \\
(1-6)\end{array}$ & $\begin{array}{l}11 \\
(5-21)\end{array}$ & $\begin{array}{l}3598 \\
(56 \cdot 6 \%)\end{array}$ & $793(12 \cdot 5 \%)$ \\
\hline
\end{tabular}

in south Asia and the highest rates were reported in east and southeast Asia and the Middle East (table 3). Of the patients with sepsis, $1808(60 \cdot 8 \%)$ already had sepsis on admission to the ICU, and 1681 (56.5\%) had septic shock.

ICU and hospital mortality rates varied widely by geographical region (table 3). Crude ICU and hospital mortality rates were higher in patients admitted to ICUs in upper-middle income countries than to ICUs in low and lower-middle or high-income countries (all $\mathrm{p}<0 \cdot 0001)$. The highest crude ICU and hospital mortality rates were recorded in patients admitted to ICUs in countries with upper-middle GNI (table 3). Hospital mortality rates per country according to GNI are shown in the appendix.

ICU and hospital mortality rates in patients with sepsis were $25 \cdot 8 \%(24 \cdot 2-27 \cdot 4)$ and $35 \cdot 3 \% \quad(33 \cdot 5-37 \cdot 1)$, respectively, and varied between $11.9 \%$ and $19.3 \%$ (Oceania) to $39.5 \%$ and $47.2 \%$ (Africa), respectively.

The unconditional model suggested significant betweencountry variations $(\operatorname{var}=0 \cdot 19, \mathrm{p}=0 \cdot 002)$ and betweenhospital variations (var $=0.43, \mathrm{p}<0 \cdot 0001$ ) in the individual risk of in-hospital death (appendix). Between-hospital variations seemed to be greater than between-country variations, as shown by the median OR (1.86 vs 1.51).

After controlling for patient and hospital factors and GNI (country factor), the differences across hospitals decreased by $49 \%$ but remained significant (var $=0 \cdot 34$, $\mathrm{p}<0 \cdot 0001)$; by contrast, the differences across countries disappeared after adjustment $(82 \%$ decrease, var $=0.03$, $\mathrm{p}=0 \cdot 18)$. There was a stepwise increase in the adjusted risk of in-hospital death with decreasing GNI (figure, appendix) such that, compared with patients from high income countries, those from upper-middle income countries (OR 1.74, 95\% CI 1.38-2.20) and low and lower-middle income countries (OR 2.10, 1.46-3.03) had a greater risk of in-hospital death.

Patients with sepsis were more at risk of in-hospital death than those without (OR 1.29, 1.13-1.48). Other independent risk factors for in-hospital death included older age, higher SAPS II score, medical or trauma admission (compared with the surgical admission group), admissions from the hospital floor (compared with admissions from the emergency room or ambulance), comorbid cancer, chronic heart failure (NYHA III/IV), 


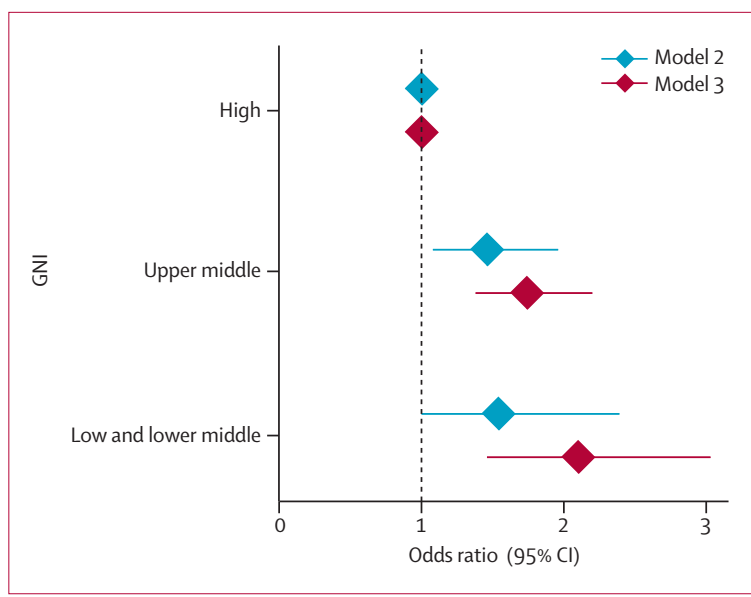

Figure: Adjusted odds ratios of in-hospital death

Odds ratios are according to the $\mathrm{GNI}$ in the whole cohort, with patients admitted to intensive care units in countries with high GNI as the reference category. Model 2 includes adjustment for hospital-level variables. Model 3 includes adjustment for patient-level and hospital-level variables. $\mathrm{GNI}=$ gross national income.

immunosuppression, cirrhosis, and the need for mechanical ventilation or renal replacement therapy (appendix).

\section{Discussion}

Our study shows important aspects related to the burden of intensive care worldwide. Notably, after adjustment for possible confounders in a multivariable analysis, there was a stepwise increase in the risk of in-hospital death according to decreasing GNI. There are many possible reasons for this finding, including potential issues related to differences in availability of trained staff and treatments or in quality of care. There are few data available concerning intensive care facilities in lower income countries (panel). ${ }^{14} \mathrm{~A}$ recent study from Tanzania reported that although sufficient equipment and drugs seemed to be available for emergency and critical care, the infrastructure, training, and process of care were inadequate. ${ }^{16}$ Similar findings have been reported from other low income countries. ${ }^{17-20}$

Using multilevel modelling to assess the reasons involved in the individual risk of in-hospital death, our findings suggest that the centre effect might be more important than the effect of GNI, suggesting that differences in ICU organisation among centres within any one country have a key role in determining patient outcomes. Various organisational issues have been shown to affect ICU patient outcomes in different countries. ${ }^{21-23} \mathrm{In}$ a study of 24 ICUs in one US county, ${ }^{24}$ patients with acute lung injury had better outcomes if cared for in a closedformat (units that transferred all patients to an intensive care team or where a consultation with an intensivist who then shared responsibility for patient management with the admitting physician was mandatory) than in an openformat (units where any attending physician with ICU admitting privileges could be responsible for patient management) ICU. In a recent study of 69 ICUs across the
$\mathrm{USA}^{23}$ daily care review and a lower bed-to-nurse ratio were associated with a lower annual ICU mortality, but not closed ICU format or 24 h presence of an intensivist. In an analysis of the large EPIC II database, a high nurse-topatient ratio was noted to be independently associated with a lower risk of in-hospital death (Sakr Y, unpublished). Availability of a consultant-level intensivist and use of multidisciplinary clinical wardrounds are known to be associated with a high level of quality of care. ${ }^{25}$ The effect of ICU infrastructure, staff training and availability, and process of care on patient outcomes clearly needs further study so that intensive care provision can be optimised across centres and resources can be targeted most appropriately on a global basis.

Our study has several limitations that should be considered when interpreting the data. First, although the audit included many ICUs, the voluntary nature of the participation might have affected the number and types of centres participating, perhaps particularly in the low and lower-middle income countries. This might have led to an underestimation of the burden of critical illness in these areas. Moreover, we are unable to assess how representative the participating hospitals are of their region. For example, a high percentage of the ICUs from low and lower-middle income countries reported 24-h intensivist cover and many reported high availability of ancillary staff, which seems to conflict with some other reports from these countries. ${ }^{14}$ Some patient characteristics also seem to conflict with other data from these regions-eg, the rate of HIV infection was lowest in the low and lower-middle income countries, although in general the prevalence of HIV in these countries, many of which are located in sub-Saharan Africa, is particularly high. ${ }^{14}$ The reasons for these apparent differences are not clear but probably relate to, at least partly, some degree of sample bias. The lower prevalence of reported comorbidities (COPD and heart failure) in low and lower-middle income countries versus high income countries might have been related to reporting bias. With the likely lower access to medical facilities in low and lower-income countries, it is possible that patients are less likely to have been diagnosed with a chronic disease. Nonetheless, our cohort provides largescale comparative data in critically ill patients across multiple geographical areas and should be regarded as a unique initiative that can encourage future international collaboration in this field.

Second, data collection was not monitored and only incongruous data were verified. Third, missing SOFA scores were imputed by linear interpolation or carrying values backward or forward, which might potentially affect our estimations; however, the percentage of imputed data was small (about 3\%) so it is unlikely that this will have had a major influence. Fourth, we analysed countries according to GNI, rather than the percentage of GDP allocated to health care specifically, but these data are difficult to obtain and less comparable because their 


\section{Panel: Research in context}

\section{Systematic review}

We searched PubMed for reports published before Dec 1 , 2013, with the search terms "critical illness", "intensive care medicine", "burden", "outcome", and "global". The search was limited to reports in English. We also checked the reference lists of reports identified in the search. Global comparative cohorts investigating intensive care practice, outcome, and the burden of critical illness are lacking. Several recent papers have highlighted the lack of information on the global burden of critical illness and availability of intensive care and called for studies to broaden knowledge in this field., 2,14,15

\section{Interpretation}

To our knowledge, our study provides the largest available report of information related to provision of intensive care worldwide. The results of the present audit show a strong relation between the risk of death and the global national income, and suggest that differences in ICU organisation among centres play an important part in determining risk of death. Our data also show that sepsis remains a major health problem worldwide, associated with high mortality rates in all countries.

definition varies among countries. Fifth, because of the study design, data were collected over a short period of time and it is possible that this period was not representative of the average annual situation in each centre. Finally, the results of the multilevel analysis might not have accounted for unmeasured variables, but we adjusted for a large number of variables that might affect outcome.

The frequency of sepsis in our cohort was similar to that reported in the SOAP study $(37 \cdot 4 \%){ }^{26}$ Other studies ${ }^{27-29}$ have reported lower incidences of sepsis, but our study did not include routine postoperative patients.

Although study entry was entirely voluntary, the large amount of data collected on more than 10000 patients from more than 80 countries shows the perceived need for such an international audit. International epidemiological data such as these provide a valuable insight into the global burden of critical illness worldwide. Indeed, several recent reports have highlighted the lack of information on the global burden of critical illness and availability of intensive care and called for studies to broaden knowledge in this field. ${ }^{2,14,15}$ The results of the present audit, bearing in mind the limitations of the study design as discussed, show a strong relation between the risk of death and the GNI, and suggest that differences in ICU organisation among centres might have an important role in determining risk of death, although our data are insufficient to capture which specific aspects might be involved. Further study is needed to better define those aspects of ICU organisation that have the greatest effect.

Our data also show that sepsis remains a major health problem worldwide, associated with high mortality rates in all countries, supporting the need for continued emphasis to be placed on the epidemiology, prevention, and treatment of this important societal problem.

\section{Contributors}

J-LV conceived the study. J-LV, JCM, KR, MA, HN, EJ, and YS designed the study. SAN-S, BF, IM-L, JL, and PP were involved in acquiring data $\mathrm{HN}$ and YS were responsible for the statistical analysis. HN, YS, and J-LV interpreted the data. YS and J-LV wrote the first draft of the report. JCM, SAN-S, BF, IM-L, JL, KR, MA, PP, HN, and EJ revised the report critically for important intellectual content. All authors approved the final version of the manuscript.

\section{Declaration of interests}

We declare that we have no competing interests.

\section{References}

1 Halpern NA, Pastores SM. Critical care medicine in the United States 2000-2005: an analysis of bed numbers, occupancy rates, payer mix, and costs. Crit Care Med 2010; 38: 65-71.

2 Adhikari NK, Fowler RA, Bhagwanjee S, Rubenfeld GD. Critical care and the global burden of critical illness in adults. Lancet 2010; 376: $1339-46$.

3 Le Gall JR, Lemeshow S, Saulnier F. A new Simplified Acute Physiology Score (SAPS II) based on a European/North American multicenter study. JAMA 1993; 270: 2957-63.

4 Knaus WA, Draper EA, Wagner DP, Zimmerman JE. APACHE II: a severity of disease classification system. Crit Care Med 1985; 13: 818-29.

5 Vincent JL, Moreno R, Takala J, et al. The SOFA (Sepsis-related Organ Failure Assessment) score to describe organ dysfunction/ failure. On behalf of the Working Group on Sepsis-Related Problems of the European Society of Intensive Care Medicine. Intensive Care Med 1996; 22: 707-10.

6 Calandra T, Cohen J. The international sepsis forum consensus conference on definitions of infection in the intensive care unit Crit Care Med 2005; 33: 1538-48.

7 Vincent JL, Opal SM, Marshall JC, Tracey KJ. Sepsis definitions: time for change. Lancet 2013; 381: 774-75.

8 World Bank. GNI per capita, Atlas method (current US\$). http://data.worldbank.org/indicator/NY.GNP.PCAP.CD (accessed March 10, 2014).

9 Agresti A, Coull BA. Approximate Is better than "exact" for interval estimation of binomial proportions. Am Stat 1998 52: 119-26.

10 Larsen K, Petersen JH, Budtz-Jorgensen E, Endahl L. Interpreting parameters in the logistic regression model with random effects. Biometrics 2000; 56: 909-14.

11 Larsen K, Merlo J. Appropriate assessment of neighborhood effects on individual health: integrating random and fixed effects in multilevel logistic regression. Am J Epidemiol 2005; 161: 81-88.

12 Merlo J, Chaix B, Ohlsson H, et al. A brief conceptual tutoria of multilevel analysis in social epidemiology: using measures of clustering in multilevel logistic regression to investigate contextual phenomena. J Epidemiol Community Health 2006; 60: 290-97.

13 Rasbash J, Steele F, Browne W, Goldstein H. A user's guide to MLwiN. Bristol: University of Bristol, 2012.

14 Dunser MW, Baelani I, Ganbold L. A review and analysis of intensive care medicine in the least developed countries. Crit Care Med 2006; 34: 1234-42.

15 Fowler RA, Adhikari NK, Bhagwanjee S. Clinical review: critical care in the global context-disparities in burden of illness, access, and economics. Crit Care 2008; 12: 225.

16 Baker T, Lugazia E, Eriksen J, Mwafongo V, Irestedt L, Konrad D. Emergency and critical care services in Tanzania: a survey of ten hospitals. BMC Health Serv Res 2013; 13: 140.

17 Hsia RY, Mbembati NA, Macfarlane S, Kruk ME. Access to emergency and surgical care in sub-Saharan Africa: the infrastructure gap. Health Policy Plan 2012; 27: 234-44.

18 Jochberger S, Ismailova F, Lederer W, et al. Anesthesia and its allied disciplines in the developing world: a nationwide survey of the Republic of Zambia. Anesth Analg 2008; 106: 942-48. 
19 Baelani I, Jochberger S, Laimer T, et al. Availability of critical care resources to treat patients with severe sepsis or septic shock in Africa: a self-reported, continent-wide survey of anaesthesia providers. Crit Care 2011; 15: R10.

20 Bataar O, Lundeg G, Tsenddorj G, et al. Nationwide survey on resource availability for implementing current sepsis guidelines in Mongolia. Bull World Health Organ 2010; 88: 839-46.

21 Durairaj L, Torner JC, Chrischilles EA, Vaughan Sarrazin MS Yankey J, Rosenthal GE. Hospital volume-outcome relationships among medical admissions to ICUs. Chest 2005; 128: 1682-89.

22 van der Sluis FJ, Slagt C, Liebman B, Beute J, Mulder JW, Engel AF. The impact of open versus closed format ICU admission practices on the outcome of high risk surgical patients: a cohort analysis. BMC Surg 2011; 11: 18.

23 Checkley W, Martin GS, Brown SM, et al. Structure, process, and annual ICU mortality across 69 centers: United States Critical Illness and Injury Trials Group Critical Illness Outcomes Study. Crit Care Med 2014; 42: 344-56.

24 Treggiari MM, Martin DP, Yanez ND, Caldwell E, Hudson LD, Rubenfeld GD. Effect of intensive care unit organizational model and structure on outcomes in patients with acute lung injury. Am J Respir Crit Care Med 2007; 176: 685-90.
25 Rhodes A, Moreno RP, Azoulay E, et al. Prospectively defined indicators to improve the safety and quality of care for critically ill patients: a report from the Task Force on Safety and Quality of the European Society of Intensive Care Medicine (ESICM). Intensive Care Med 2012; 38: 598-605.

26 Vincent JL, Sakr Y, Sprung CL, et al. Sepsis in European intensive care units: results of the SOAP study. Crit Care Med 2006; 34: 344-53.

27 Brun-Buisson C, Meshaka P, Pinton P, Vallet B. EPISEPSIS: a reappraisal of the epidemiology and outcome of severe sepsis in French intensive care units. Intensive Care Med 2004; 30: 580-88.

28 Padkin A, Goldfrad C, Brady AR, Young D, Black N, Rowan K. Epidemiology of severe sepsis occurring in the first $24 \mathrm{hrs}$ in intensive care units in England, Wales, and Northern Ireland. Crit Care Med 2003; 31: 2332-38.

29 Sakr Y, Elia C, Mascia L, et al. Epidemiology and outcome of sepsis syndromes in Italian ICUs: a regional multicenter observational cohort. Minerva Anestesiol 2013; 79: 993-1002. 